\title{
How can we optimize the use of prostate cancer registries?
}

\author{
"...efforts should be made to improve the quality of data collected, to \\ identify standard sets of disease-specific variables that include \\ patient-reported validated end points, and to implement the collection \\ of liquid and tissue samples from patients with prostate cancer."
}

\author{
Giorgio Gandaglia ${ }^{*, 1,2}$, Nicola Fossati ${ }^{1,2}$, Francesco Montorsi ${ }^{1,2}$ \\ \& Alberto Briganti ${ }^{1,2}$
}

First draft submitted: 3 January 2016; Accepted for publication: 3 February 2016; Published online: 1 March 2016

\begin{abstract}
In the evidence-based medicine era where the highest level of evidence is provided by randomized controlled trials, the relevance and generalizability of information coming from retrospective studies is often questioned [1]. Nonetheless, investigations relying on cancer registries, which are organized systems that collect data from a specific population affected by a particular disease [2], can often provide unique information that allows clinicians to understand the natural history of the disease itself, ultimately improving its clinical management. Although randomized controlled trials will continue to provide the highest level of evidence in the future, they might be limited by poor accrual, relatively short follow-up intervals and inclusion of highly selected cohorts that may limit the generalizability of their findings [1,3-4]. For example, elderly and sicker patients are often excluded by these trials. This is particularly important in the setting of prostate cancer ( $\mathrm{PCa}$ ) given its increasing incidence with age and the relatively prolonged natural history of the disease itself [5]. Studies based on registries might in part circumvent these limitations, where the inclusion of large cohorts of men
\end{abstract}

treated at the community level, as well as long follow-up intervals, might provide a valid data source to give answers to clinically relevant questions [2-4]. Therefore, findings obtained in the context of cancer registries should not be entirely disputed but rather integrated with observations coming from randomized controlled trials to achieve high-level clinically relevant evidence [4].

\section{Types of cancer registries: population-based versus prospective patient registries \\ Several registries are currently available in $\mathrm{PCa}$ [2]. These registries can be classified in two main categories: population-based versus prospective patient registries. The term population-based registry refers to the systematic collection of data on all patients with a certain disease within a determined population or geographic area [2]. Typical examples of population-based registries in the field of $\mathrm{PCa}$ are represented by the Surveillance, Epidemiology, and End Results (SEER), the SEER-Medicare and the Prostate Cancer data Base Sweden (PCBaSe) databases [2,6]. These registries}

\section{KEYWORDS}

- biomarkers $\bullet$ databases $\bullet$ outcomes

- prostate cancer $\bullet$ registries 
“...both population-based and prospective patient prostate cancer registries currently provide unique information that can be used to advance our knowledge of the disease itself." include large cohorts of men with PCa and detailed demographic and disease characteristics, as well as follow-up data. Nonetheless, they were not designed for research purposes and they typically rely on administrative codes that might be in some form incomplete or inaccurate $[1,2]$. On the contrary, prospective patient registries are specifically developed to collect detailed $\mathrm{PCa}$-specific information defined a priori on diagnosis, treatment and follow-up [2].

\section{Current uses of cancer registries in $\mathrm{PCa}$}

Large population-based PCa registries are typically used to provide data on the incidence, prevalence and mortality of a specific disease. This is the case, for example, of the SEER database, which reports annually detailed information on the number of patients diagnosed with PCa and on those who died from it in the USA [7]. Population-based and prospective diseasespecific registries can also provide insight on patient and disease characteristics at the time of diagnosis, trends of adoption of novel therapies, and disparities in the treatment of PCa according to age, geographical distribution, race and sociodemographic status [8]. Similarly, they might be used to assess adherence to clinical guidelines and evidence-based recommendations, where a disappointingly low degree of adoption of guidelines has been recently reported in Urology $[9,10]$. Investigators can also use these data-sources to assess oncologic and quality of life outcomes after the administration of specific therapies. However, the nature of population-based registries, which are typically based on administrative claims, precludes availability of details on disease recurrence and validated patient-reported quality of life data [1,2]. For these reasons, caution is needed in the interpretation of results coming from studies using these registries to compare effectiveness of different treatments. Such retrospective comparisons are even more biased given the impossibility to account for factors that may have influenced patient selection and the entire clinical decision making process. Conversely, prospective disease-specific registries such as the UCSF Cancer of the Prostate Strategic Urologic Research Endeavor (CaPSURE), Michigan Urological Survey Improvement Collaborative (MUSIC), South Australian Prostate Cancer Clinical Outcome Collaborative (SA-PCCOC), and others are able to comprehensively capture detailed information on biochemical recurrence, clinical progression, disease-specific mortality and functional outcomes such as bowel function, urinary continence and erectile function after treatment [2]. The main advantages of cancer registries in this setting as compared with randomized trials, are that randomized trials rely on the availability of cohorts of patients representing the 'real-life' scenario, relatively long follow-up periods and large sample size. In addition, these data sources contain information on healthcare expenditures and costs. This often allows investigators for a careful analysis of the cost-benefit ratio associated with the adoption of a specific treatment in a certain healthcare setting [2,11-13]. Finally, registries are able to provide feedback to physicians and hospitals regarding their performance, adherence to guidelines, expenditures and costs and validated outcomes at short- and long-term follow-up [2,14-15]. The comparison of individual results to what observed at a community or population level might motivate healthcare providers to promote specific changes and to implement quality improvement programs [14]. Similarly, these data sources might be used to identify areas where efforts should be made in order to improve the quality of care and individual performances.

\section{Future perspective}

Cancer registries will evolve in the next few years and several improvements are expected. For example, the implementation of electronic online databases will allow for the automatic streaming of relevant data to these registries. Moreover, the ideal registry should also be able to provide high-quality data prospectively recorded with the identification of the variables of interest and end points 'a priori'. Validated patient-reported outcome measures on quality of life after treatment should also be included [16] . In this context, Martin et al. [16] recently defined a set of outcomes that should be recorded in patients with clinically localized PCa to even their assessment, expand interpretability of comparison between different cohorts, and, ultimately, provide clinically relevant information that might improve patient outcomes. Another relevant issue regards the quality of data collected by registries. Indeed, inaccurate and incomplete coding, as well as errors during the process of data collection, might limit the validity of studies performed in this setting. Therefore, quality control initiatives that include the involvement of trained personnel, periodical control of the data collected and 
validation studies should be implemented [17-19]. Finally, in the next few years the evaluation of standard clinical and pathologic disease characteristics might not be sufficient to provide information that advances our knowledge in the setting of PCa. Indeed, biomarkers will play an important role in the risk stratification, treatment planning and decision-making process in men with PCa in an era of personalized medicine [20]. Collection of serum, urine and tissue samples would allow for assessing the role of biological and genetic prognostic markers and for validating novel risk stratification tools based on these parameters. Due to the nature of cancer registries, these studies would benefit from the inclusion of large and unselected cohorts where different ethnicity and socio-demographic situations are well represented.

In conclusion, both population-based and prospective patient $\mathrm{PCa}$ registries currently provide unique information that can be used to advance our knowledge of the disease itself. Nonetheless, efforts should be made to improve the quality of data collected, to identify standard sets of disease-specific variables that include patientreported validated end points, and to implement the collection of liquid and tissue samples from patients with PCa. Information coming from registries will continue to play a major role in the near future especially if they will be used to optimize the design and methodology of randomized prospective trials, thus creating a continuum between different levels of evidence.

\section{Financial \& competing interests disclosure}

The authors have no relevant affliations or financial involvement with any organization or entity with a financial interest in or financial conflict with the subject matter or materials discussed in the manuscript. This includes employment, consultancies, honoraria, stock ownership or options, expert testimony, grants or patents received or pending, or royalties.

No writing assistance was utilized in the production of this manuscript.

\section{References}

1 Booth CM, Tannock IF. Randomised controlled trials and population-based observational research: partners in the evolution of medical evidence. Br. J. Cancer 110(3), 551-555 (2014).

2 Gandaglia G, Bray F, Cooperberg MR et al. Prostate cancer registries: current status and future directions. Eur. Urol. doi:10.1016/j. eururo.2015.05.046 (2015) (Epub ahead of print).

3 Dahm P, N'Dow J, Holmberg L, Hamdy F. The future of randomised controlled trials in urology. Eur. Urol. 66(1), 1-3 (2014).

4 Gandaglia G, Bray F, Cooperberg MR et al. Reply from authors re: Julia Verne, Luke Hounsome, Roger Kockelbergh, Jem Rashbass. Improving outcomes from prostate cancer: unlocking the treasure trove of information in cancer registries. Eur. Urol. doi:10.1016/j.eururo.2015.09.036 (2015) (Epub ahead of print).

5 Briganti A, Karnes RJ, Gandaglia G et al. Natural history of surgically treated high-risk prostate cancer. Urol. Oncol. 33(4), 163. e167-113 (2015).

6 Hussein AA, Welty CJ, Broering J, Cooperberg MR, Carroll PR. Review article: national prostate cancer registries: contemporary trends of prostate cancer in the United States. Urol. Pract. 1, 198-204 (2014).
7 Siegel RL, Miller KD, Jemal A. Cancer statistics, 2015. CA Cancer J. Clin. 65(1), 5-29 (2015).

8 Mahal BA, Ziehr DR, Aizer AA et al. Getting back to equal: the influence of insurance status on racial disparities in the treatment of African American men with high-risk prostate cancer. Urol. Oncol. 32(8), 1285-1291 (2014).

9 Choi WW, Williams SB, Gu X, Lipsitz SR, Nguyen PL, Hu JC. Overuse of imaging for staging low risk prostate cancer. J. Urol. 185(5), 1645-1649 (2011).

10 Makarov DV. Regional variations in US cancer imaging data: a warning for imaging overuse. Future Oncol. 11(22), 3057-3060 (2015).

11 Gandaglia G, Sammon JD, Chang SL et al. Comparative effectiveness of robot-assisted and open radical prostatectomy in the postdissemination era. J. Clin. Oncol. 32(14), 1419-1426 (2014).

12 Gandaglia G, Montorsi F, Karakiewicz PI, Sun M. Robot-assisted radical prostatectomy in prostate cancer. Future Oncol. 11(20), 2767-2773 (2015).

13 Jacobs BL, Zhang Y, Schroeck FR et al. Use of advanced treatment technologies among men at low risk of dying from prostate cancer. JAMA 309(24), 2587-2595 (2013).

14 Cooperberg MR. Progress in management of low-risk prostate cancer: how registries may change the world. Eur. Urol. 67(1), 51-52 (2015).

15 Womble PR, Montie JE, Ye Z et al. Contemporary use of initial active surveillance among men in Michigan with low-risk prostate cancer. Eur. Urol. 67(1), 44-50 (2015).

16 Martin NE, Massey L, Stowell C et al. Defining a standard set of patient-centered outcomes for men with localized prostate cancer. Eur. Urol. 67(3), 460-467 (2015).

17 Barocas DA, Chen V, Cooperberg M et al. Using a population-based observational cohort study to address difficult comparative effectiveness research questions: the CEASAR study. J. Comp. Eff. Res. 2(4), 445-460 (2013).

18 Porten SP, Cooperberg MR, Konety BR, Carroll PR. The example of CaPSURE: lessons learned from a national disease registry. World J. Urol. 29(3), 265-271 (2011).

19 Scosyrev E, Messing J, Noyes K, Veazie P, Messing E. Surveillance Epidemiology and End Results (SEER) program and populationbased research in urologic oncology: an overview. Urol. Oncol. 30 (2), 126-132 (2012).

20 Bostrom PJ, Bjartell AS, Catto JW et al. Genomic predictors of outcome in prostate cancer. Eur. Urol. 68(6), 1033-1044 (2015). 